Annuaire suisse de politique de développement

$14 \mid 1995$

Annuaire Suisse - Tiers Monde 1995

\title{
Cohérence des relations entre la Suisse et l'Indonésie
}

Konrad Specker et Hans Peter Maag

\section{(2) OpenEdition}

1 Journals

Édition électronique

URL : http://journals.openedition.org/aspd/1453

DOI : $10.4000 /$ aspd. 1453

ISSN : 1663-9669

Éditeur

Institut de hautes études internationales et du développement

Édition imprimée

Date de publication : 1 février 1995

Pagination : 182-185

ISSN : 1660-5934

\section{Référence électronique}

Konrad Specker et Hans Peter Maag, "Cohérence des relations entre la Suisse et l'Indonésie ",

Annuaire suisse de politique de développement [En ligne], 14 | 1995, mis en ligne le 03 mai 2013, consulté le 08 septembre 2020. URL : http://journals.openedition.org/aspd/1453 ; DOI : https:// doi.org/10.4000/aspd. 1453 


\title{
Cohérence des relations entre la Suisse et l'Indonésie
}

\author{
Konrad Specker, Hans Peter Maag, Section Asie II, DDA
}

\section{Evolution du programme de la DDA en Indonésie}

Au cours des 25 dernières années, l'Indonésie a obtenu des succès considérables en matière de développement. Le pays enregistre des taux de croissance supérieurs à $6 \%$ et le pourcentage de population vivant au niveau de pauvreté absolue a été réduit de 60 à 15\%. En 1967, I'Indonésie faisait encore partie, avec un revenu par tête d'habitant de 60 US\$, des pays les plus pauvres du monde. Aujourd'hui, avec un revenu par tête d'habitant d'environ 650 US\$, le pays se retrouve au seuil de la catégorie des pays à revenu moyen.

Depuis le début de la coopération bilatérale au développement entre la Suisse et l'Indonésie, dans la seconde moitié des années 60 , l'Indonésie fait partie des pays principaux bénéficiaires de la coopération au développement assurée par la DDA. La formation professionnelle technique, le développement urbain et le développement rural sont devenus les principaux secteurs de la coopération. Le volume financier annuel moyen se situe dans la première moitié des années 90 dans les 20 mio. de francs suisses. 
La composition des instruments de la DDA et de l'OFAEE utilisés dans le cadre de la coopération bilatérale au développement est fonction du stade de développement du pays partenaire et suit son évolution. Selon la pratique courante, la DDA se concentre avec sa politique de "pays principaux bénéficiaires" sur la catégorie des pays en développement les plus pauvres. Les pays à faible revenu par tête d'habitant (300 à 600 US\$) relèvent de la compétence aussi bien de la DDA que de l'OFAEE. Dans la catégorie des pays à revenu moyen, la composition - conformément à l'adage "from aid to trade» - penche en faveur d'instruments de l'OFAEE.

Tenant compte de l'état de développement de l'Indonésie et de sa situation macro-économique, la DDA se concentre actuellement sur une transformation du programme indonésien. En conséquence, la coopération technique traditionnelle sera progressivement réduite. Dans le même temps, l'OFAEE étudie la possibilité d'engager de nouveaux instruments dans la perspective de l'encouragement à l'investissement et du transfert des technologies. Cette transformation implique une réorientation des relations helvetico-indonésiennes.

Parallèlement à la réduction des activités en cours, la DDA lancera un programme résiduel restreint qui a pour but:

- de poursuivre la consolidation des acquis à ce jour de la coopération;

- d'apporter une contribution à la maîtrise des problèmes de développement

(également dans une perspective régionale) qui font partie des principaux défis des prochaines années.

Ce programme se composera de trois éléments qui sont étroitement liés entre eux: i) promotion des petites et moyennes industries; ii) management du développement urbain; et iii) environnement (urbain). Parallèlement à ce programme, un crédit sera également réservé - dans le sens d'un suivi des efforts déployés et d'un entretien des relations - à des mesures de soutien ponctuelles en faveur d'institutions, dont la mise sur pied et le développement ont été soutenus dans le cadre de la coopération bilatérale au développement suisse apportée jusqu'ici.

Pour lancer ce programme, on recherche un maximum de synergies avec d'autres acteurs des relations helvético-indonésiennes (à savoir, avec I'OFAEE). Dans le domaine PME et environnement, par ex., il pourrait y avoir des synergies dans les domaines où les mesures de soutien profitent à des entreprises indonésiennes dynamiques dotées d'un potentiel d'emploi élevé et offrant la possibilité d'introduire des méthodes de production ménageant l'environnement.

\section{Problèmes de cohérence}

Dans l'optique de la politique de développement, la question de la cohérence se pose non seulement par rapport aux acteurs de la politique (économique) étrangère de la Suisse, mais aussi à l'égard des conflits entre objectifs, inhérents au développement indonésien lui-même. 
Au cours du sixème plan quinquennal (1994/95-1998/99), des taux de croissance de $6 \%$ seront nécessaires pour maintenir la stabilité macro-économique et atteindre les objectifs fixés en matière de création d'emplois et de lutte contre la pauvreté, ainsi que dans le domaine de la suppression du déficit de la balance des paiements et de la dette extérieure.

La clef de la croissance économique réside dans une accélération de l'industrialisation et dans le développement des exportations de produits industriels. Cependant, l'industrialisation se fait au prix d'une destruction alarmante de l'environnement et d'une recrudescence des conflits du travail. En même temps, l'Indonésie se voit exposée à une concurrence croissante de la part d'autres pays asiatiques employant une main-d'oeuvre bon marché. Tout compte fait, le développement entraîne aussi des inégalités régionales et sociales.

C'est pourquoi l'Indonésie est confrontée à une intensification des champs de tension économiques, sociaux et écologiques. Si l'Indonésie ne parvient pas à protéger efficacement son environnement, à absorber une classe ouvrière en pleine expansion, à accorder à cette dernière les droits fondamentaux et à lui garantir de meilleures conditions, à obtenir une plus large participation aux fruits du développement et à entamer un réel processus d'ouverture politique, il faut s'attendre à moyen et à long terme à une déstabilisation qui remettra fondamentalement en cause la durabilité du développement.

Du point de vue de la politique de développement, on peut se demander, pour ce qui concerne le problème de la cohérence, si les relations helvéticoindonésiennes dans leur ensemble et les activités des divers acteurs en particulier favoriseront ou contrecarreront la maîtrise de ces champs de tension autrement dit: la promotion d'un développement plus équilibré et plus large, la protection de l'environnement et l'amélioration des conditions de travail.

Vu l'importance potentiellement croissante de l'Indonésie en tant que partenaire économique, la question suivante se pose par analogie: les intérêts économiques et de politique économique de la Suisse peuvent-ils être sauvegardés de telle sorte qu'il Isoit possible de contribuer en Indonésie à surmonter la "monopolisation", à développer le marché intérieur, à promouvoir des PME dotées d'un grand potentiel d'emplois et à protéger l'environnement?

Dans le cadre d'une telle contribution, on peut parfaitement exercer un effet bénéfique en matière de bonne gestion gouvernementale et de droits de l'homme. Une approche constructive est préférable à des conditionnalités. I'Indonésie accepte le dialogue bilatéral en matière de bonne gestion gouvernementale et de droits de l'homme, mais rejette les conditionnalités. La Suisse recourt à la voie diplomatique et elle est intervenue à diverses occasions auprès du gouvernement indonésien. Par rapport au problème de la cohérence se pose alors la question de savoir si, le cas échéant, les différents aspects des relations économiques, commerciales et de politique de développement de la Suisse avec l'Indonésie contrecarrent ce dialogue, si ces relations peuvent ou doivent être subordonnées aux intérêts que présente ce dialogue et selon quels critères. 


\section{Conclusion}

Dans le cas de l'Indonésie, la cohérence dans les relations de politique étrangère et d'économie extérieure peut être évaluée en priorité du point de vue des impératifs du développement national et des conflits entre objectifs. Par conséquent, on peut atteindre une cohérence si les diverses activités - qu'elles soient ou non coordonnées - favorisent un développement socialement et écologiquement viable ou tout au moins ne l'entravent pas. Si un tel développement équilibré et ainsi conçu est encouragé, il est possible de réaliser les objectifs stratégiques de notre politique étrangère.

Des mécanismes institutionnels qui favorisent le dialogue entre les acteurs des relations helvético-indonésiennes peuvent sûrement contribuer à la mise en pratique des objectifs donnés par la ligne directrice. Cela dit, une question demeure: à quel point de tels mécanismes doivent et/ou peuvent-ils avoir un caractère obligatoire? Le point décisif pour la question de la cohérence est finalement la manière de gérer les contradictions qui peuvent apparaître entre les divers acteurs au niveau opérationnel. Les intérêts conflictuels et les contradictions sont inhérents à une vie économique et sociale aux multiples aspects et ne peuvent donc pas être complètement éliminés, quel que soit le niveau de cohérence de la politique suivie.

Au bout du compte, le degré de cohérence dépendra effectivement de la manière dont s'imposera au plus grand nombre la conviction selon laquelle un climat commercial et d'investissement favorable ne pourra être maintenu à moyen et à long terme qu'à la seule condition que le niveau de vie de la population s'améliore et que les différences sociales soient aplanies, l'environnement efficacement protégé, l'Etat de droit, la paix et la sécurité garantis. 